\title{
A new algorithm for neural connectivity estimation of EEG event related potentials
}

\author{
Pedro L. C. Rodrigues and Luiz A. Baccalá
}

\begin{abstract}
We propose a new algorithm for estimating neural connectivity during event related potentials (ERP) in EEG. It is composed of two steps: the estimation of a time-varying multivariate-autoregressive (MVAR) model and the calculation of the generalized partial directed coherence (gPDC) for assessing the connectivities between channels where MVAR estimation is done via an adapted version of the Nuttall-Strand algorithm, a multivariate generalization of Burg's spectral estimation algorithm. Successful algorithm validation was performed through simulations using toys model with physiologically ERP inspired features.
\end{abstract}

\section{INTRODUCTION}

Characterizing neural connectivity has become central to understanding the brain and its status under different stimulus and/or behavioural conditions [1]. So much so that it has been the focus of an almost endless number of approaches [2] with different levels of rigour, specially for the asymptotic case of multivariate single trial time series data, see [3]'s rigorous approach. Amidst the diversity of currently available approaches, those based on modeling stationary multivariate time series, such as [4], [5], [6], remain the most popular ones thanks to the wide avaliability of off-the-shelf linear modeling routines [7] and decades of practical experience.

This scenario contrasts markedly with the important case of protocols for event related data [8] which comes in the form of many repeated short lived responses rather than a single sufficiently long lasting data record for which rigorous asymptotic criteria are available. Attempts to address this issue were pioneered by [9] whose approach consisted of solving Yule-Walker equations using response averaged autocorrelation estimates. Other approaches are often described as applying the usual algorithms to 'stacked' event data whereas other ideas rely on averaging model parameters.

Our aim here is to present a multivariate autoregressive generalization of the univariate proposal in [10] (Sec. II) and examine its performance under a simulated event related scenario that induces connectivity structure switches (Sec. III ). After a brief discussion (Sec. IV), some comments finish the paper (Sec. V).

\section{MATERIAL AND METHODS}

After a brief of recap algorithm terminology (Sec. II-A) we describe details of the new algorithm and test it via simulated models that incorporate level triggered connectivity switches. Qualitative algorithm success is displayed via $g$ PDC computation [11].

The authors are with the Telecommunications and Control Department of Escola Politécnica, University of São Paulo, São Paulo, Brazil, 05508-900 prodrigues@usp.br baccala@lcs.poli.usp.br

\section{A. Estimation of a MVAR model}

Linear multivariate autoregressive modeling of simultaneously observed data $\mathbf{x}(n)=\left[x_{1}(n) x_{2}(n) \ldots x_{K}(n)\right]^{T}$ observed over $n=1, \cdots, N$ instants consists of finding the $\mathbf{A}(l)$ coefficient matrices in

$$
\mathbf{x}(n)=\sum_{l=1}^{P} \mathbf{A}(l) \mathbf{x}(n-l)+\mathbf{w}(n),
$$

where $P$ is the model order and $\mathbf{w}(n)$ stands for a zero mean Gaussian stationary innovation process with covariance matrix $\Sigma_{\mathrm{w}}$ that cannot be predicted based on past observations.

Among the many approaches [7], [12], order recursive algorithms like the Levinson-Wiggins-Robinson (LWR), Vieira-Morf (VM) and Nuttall-Strand (NS) algorithms are particularly attractive due to their reduced computational complexity and observedly robust numerical stability (mostly for VM and NS) compared to direct solutions of (1) by recasting it in terms of Yule-Walker equations [7].

In common, LWR, VM and NS, take advantage of signal stationarity to break the estimation into forward and backward prediction steps, i.e. for a given order step $p \in$ $\{1, \cdots, P\}$

$\hat{\mathbf{x}}_{p}^{f}(n)=\sum_{l=1}^{p} \mathbf{A}_{p}(l) \mathbf{x}(n-l), \quad \hat{\mathbf{x}}_{p}^{b}(n)=\sum_{l=1}^{p} \mathbf{B}_{p}(l) \mathbf{x}(n+l)$,

are respectively the best predictors that minimize the mean squared forward $\mathbf{e}_{p}^{f}(n)=\mathbf{x}(n)-\hat{\mathbf{x}}_{p}^{f}(n)$ and backward $\mathbf{e}_{p}^{b}(n)=\mathbf{x}(n)-\hat{\mathbf{x}}_{p}^{b}(n)$ prediction errors.

What distinguishes the latter algorithms is how they update coefficient matrices in going from one order to the next. In LWR, this update proceeds through

$$
\begin{aligned}
& \mathbf{A}_{p}(k)=\mathbf{A}_{p-1}(k)-\mathbf{A}_{p}(p) \mathbf{B}_{p-1}(p-k), \\
& \mathbf{B}_{p}(k)=\mathbf{B}_{p-1}(k)-\mathbf{B}_{p}(p) \mathbf{A}_{p-1}(p-k),
\end{aligned}
$$

for $k=1$ to $k=p-1$ where $\mathbf{A}_{p}(p)$ and $\mathbf{B}_{p}(p)$ are termed the forward and backward reflection coefficient matrices of order $p$ which must be computed at each algorithm order step.

In the NS algorithm, one assumes that the reflection coefficient matrices can be factored as

$$
\begin{aligned}
& \mathbf{A}_{p}(p)=\boldsymbol{\Delta}_{p}\left(\boldsymbol{\Sigma}_{p-1}^{b}\right)^{-1} \\
& \mathbf{B}_{p}(p)=\boldsymbol{\Delta}_{p}^{H}\left(\boldsymbol{\Sigma}_{p-1}^{f}\right)^{-1},
\end{aligned}
$$


where $\boldsymbol{\Sigma}_{p}^{f}$ and $\boldsymbol{\Sigma}_{p}^{b}$ are the forward and backward error covariance matrices obtained from the results in step $p-1$

$$
\begin{aligned}
& \boldsymbol{\Sigma}_{p-1}^{f}=\mathbb{E}\left[\mathbf{e}_{p-1}^{f}(n)\left(\mathbf{e}_{p-1}^{f}(n)\right)^{H}\right], \\
& \boldsymbol{\Sigma}_{p-1}^{b}=\mathbb{E}\left[\mathbf{e}_{p-1}^{b}(n)\left(\mathbf{e}_{p-1}^{b}(n)\right)^{H}\right],
\end{aligned}
$$

but where $\boldsymbol{\Delta}_{p}$ is estimated by minimizing a weighted version of the total prediction error

$$
\operatorname{trace}\left(\left(\boldsymbol{\Sigma}_{p-1}^{f}\right)^{-1} \hat{\boldsymbol{\Sigma}}_{p}^{f}+\left(\boldsymbol{\Sigma}_{p-1}^{b}\right)^{-1} \hat{\boldsymbol{\Sigma}}_{p}^{b}\right)
$$

which is equivalent to solving the following Lyapunov matrix equation:

$$
\hat{\boldsymbol{\Sigma}}_{p-1}^{f}\left(\boldsymbol{\Sigma}_{p-1}^{f}\right)^{-1} \hat{\boldsymbol{\Delta}}_{p}+\hat{\boldsymbol{\Delta}}_{p}\left(\boldsymbol{\Sigma}_{p-1}^{b}\right)^{-1} \hat{\boldsymbol{\Sigma}}_{p-1}^{b}=2 \hat{\boldsymbol{\Sigma}}_{p-1}^{f b},
$$

where $\hat{\boldsymbol{\Sigma}}_{p-1}^{f b}$ is an estimate of the cross-correlation matrix between the forward and backward prediction residuals,

$$
\boldsymbol{\Sigma}_{p-1}^{f b}=\mathbb{E}\left[\mathbf{e}_{p-1}^{f}(n)\left(\mathbf{e}_{p-1}^{b}(n-p+1)\right)^{H}\right] .
$$

Next we consider how to adapt the NS algorithm for having several realizations of short records rather than a single long process realization.

\section{B. Segment Nutall-Strand Algorithm (SegNS)}

Consider the data structure $\mathbf{x}(n, s)$, comprised of $N_{T}$ segments, each lasting $N_{s}$ time samples and assumed to obey the same model (1). The core of the algorithm consists of replacing the error covariances eqs. (7), (8) and (11) by their average over the available segments:

$$
\left\langle\hat{\boldsymbol{\Sigma}}_{p}^{(\cdot)}\right\rangle=\frac{1}{N_{T}} \sum_{s=1}^{N_{T}} \hat{\boldsymbol{\Sigma}}_{p}^{(\cdot)}(s),
$$

where the $\hat{\boldsymbol{\Sigma}}_{p}^{(\cdot)}(s)$ matrices are calculated using the forward $\left(\mathbf{e}_{p}^{f}(n, s)\right)$ and backward $\left(\mathbf{e}_{p}^{b}(n, s)\right)$ prediction errors of each segment, as in:

$$
\begin{aligned}
& \hat{\mathbf{\Sigma}}_{p}^{f}(s)=\frac{1}{N_{s}-p-1} \sum_{n=p+1}^{N_{s}} \mathbf{e}_{p}^{f}(n, s)\left(\mathbf{e}_{p}^{f}(n, s)\right)^{T}, \\
& \hat{\mathbf{\Sigma}}_{p}^{b}(s)=\frac{1}{N_{s}-p} \sum_{n=1}^{N_{s}-p} \mathbf{e}_{p}^{b}(n, s)\left(\mathbf{e}_{p}^{b}(n, s)\right)^{T}, \\
& \hat{\mathbf{\Sigma}}_{p}^{f b}(s)=\frac{1}{N_{s}-p-1} \sum_{n=p+2}^{N_{s}} \mathbf{e}_{p}^{f}(n, s)\left(\mathbf{e}_{p}^{b}(n-(p+1), s)\right)^{T} .
\end{aligned}
$$

Note that as long as segments obey the same (1) model, their lengths $N_{s}$ need not even be equal, though equality is assumed here due to the nature of ERP data.

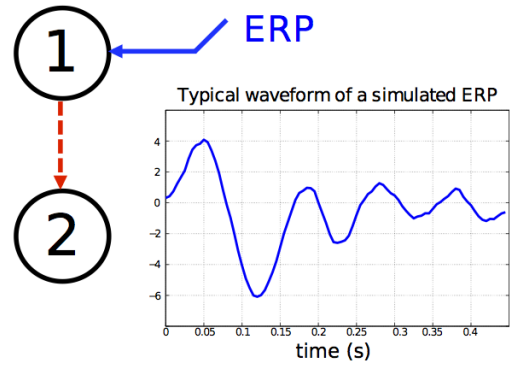

Fig. 1: Diagram depicting the network structure corresponding to the multivariate autoregressive model given by:

$\left\{\begin{aligned} x_{1}(n) & =0.95 \sqrt{2} x_{1}(n-1)-0.9025 x_{1}(n-2)+w_{1}(n) \\ x_{2}(n) & =0.95 \sqrt{2} x_{2}(n-1)-0.9025 x_{2}(n-2) \\ & +\mathbf{0 . 7 5} x_{1}(n-1)+w_{2}(n)\end{aligned}\right.$

where the boldface coefficient goes to zero in the presence of the ERP.

\section{ERP dynamics}

In actual ERP analysis situations, a number of issues need to be considered, such as model order and window size, in a tradeoff between size and goodness of fit of the MVAR model estimated with it. We adopt the guidelines presented in [9] to allow future comparison.

Assuming $N_{T}$ observed events are available, each lasting $N_{s}$ time samples, also for compatibility with [9], we subtract from each event its time mean. From each resulting record, each time data sample has its average value over events subtracted. It is the resulting data structure that is subject to SegNS processing, over $L<N_{s}$ long sliding windows, assuming an AIC model order choice criterion leading to models whose connectivity is portrayed via $g$ PDC. Unlike [9], no time-variance or ensemble-variance normalization is performed.

\section{NUMERICAL ILLUSTRATIONS}

To numerically illustrate algorithm performance, we selected two toy models. In the first one, shown in Fig. 1, the connection from $x_{1}$ towards $x_{2}$ is turned off (the coupling value $a_{21}$ goes from 0.75 to 0 ) depending on $x_{1}$ 's value, something ensured by a realistic ERP event [13] added to $x_{1}$.

The time evolution of $g$ PDC, assuming a sampling frequency of $200 \mathrm{~Hz}$, is shown on Fig. 2, where $L=12$ samples $(60 \mathrm{~ms})$ and SegNS is performed over 250 ERP trials.

The second toy model, shown in Fig. 3, has the connection from $x_{2}$ towards $x_{4}$ turned off $\left(a_{42}\right.$ goes from 0.95 to 0 ) when $x_{2}$ reaches a threshold value, due to an ERP event starting from $x_{3}$. The original network connections are re-established after $t_{\text {off }}=250 \mathrm{~ms}$. The $\mathrm{gPDC}$ timefrequency plot in Fig. 4 shows the algorithm's ability to capture connectivity change whereas a glimpse of estimation improvement with $N_{T}$ can be appreciated in Fig. 5. 


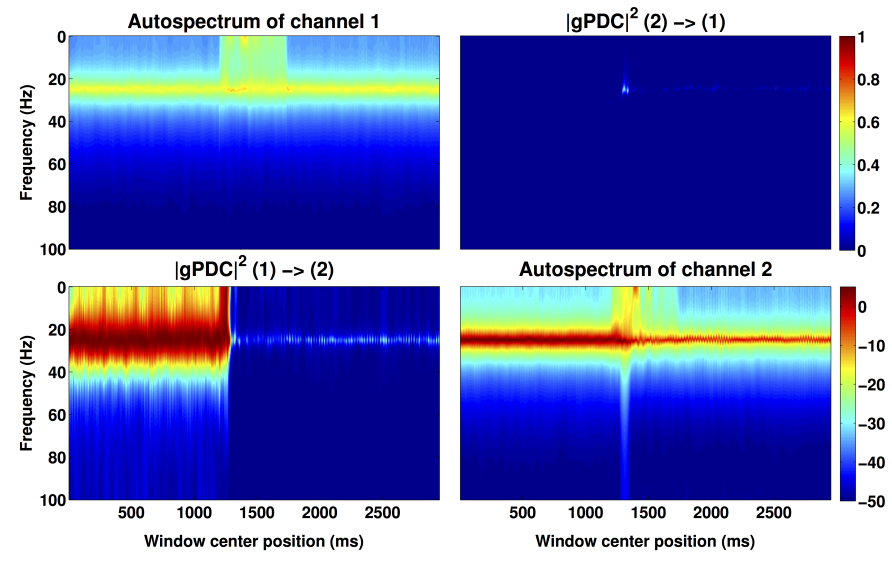

Fig. 2: Time-frequency plot of the autospectrum of each channel on the diagonals (in $\mathrm{dB}$ ). The off-diagonals show the estimated $g$ PDC over time.

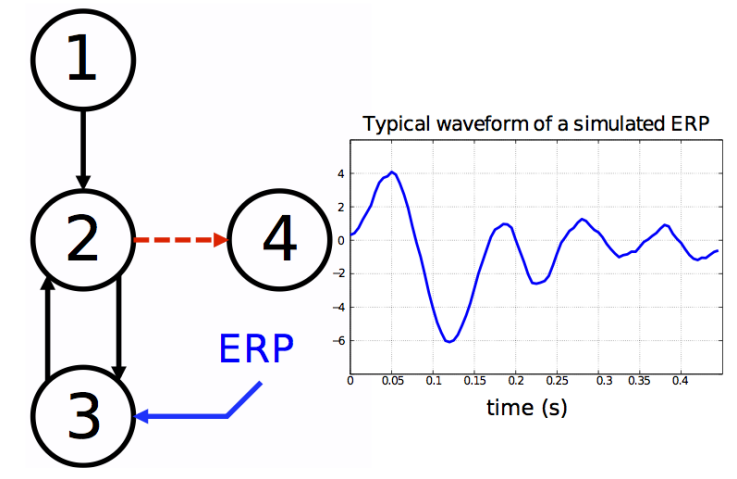

Fig. 3: Diagram depicting the network structure corresponding to the multivariate autoregressive model given by:

$$
\left\{\begin{aligned}
x_{1}(n)= & 0.95 \sqrt{2} x_{1}(n-1)-0.9025 x_{1}(n-2)+w_{1}(n) \\
x_{2}(n)= & +0.6875 \sqrt{2} x_{3}(n-1)-0.9 x_{1}(n-2) \\
& -0.4 x_{1}(n-3)+w_{2}(n) \\
x_{3}(n)= & -0.6875 \sqrt{2} x_{2}(n-1)+w_{3}(n) \\
x_{4}(n)= & 0.95 \sqrt{2} x_{4}(n-1)-0.9025 x_{4}(n-2) \\
& +0.95 x_{2}(n-1)+w_{4}(n)
\end{aligned}\right.
$$

where the boldface coefficient is zeroed upon an ERP trigger returning to its former value $t_{\text {off }}=250 \mathrm{~ms}$ later.

\section{A. Correctly dealing with segments}

One often reads treatments in the literature that propose tackling the availability of many short time records of data following the same model by constructing a single record composed of all records stacked one after the other. Then, the estimation is done using algorithms for single continuous records. Confirming [10], who warns of the danger of such procedures for the univariate case, we show that NS (red), as opposed to SegNS (blue), fails to capture the existing connection from $x_{2}$ to $x_{4}$ in the pre-ERP period. The figure also contains the theoretical results (black) with the main diagonal showing the rather biased NS (flattened) autospectra results.

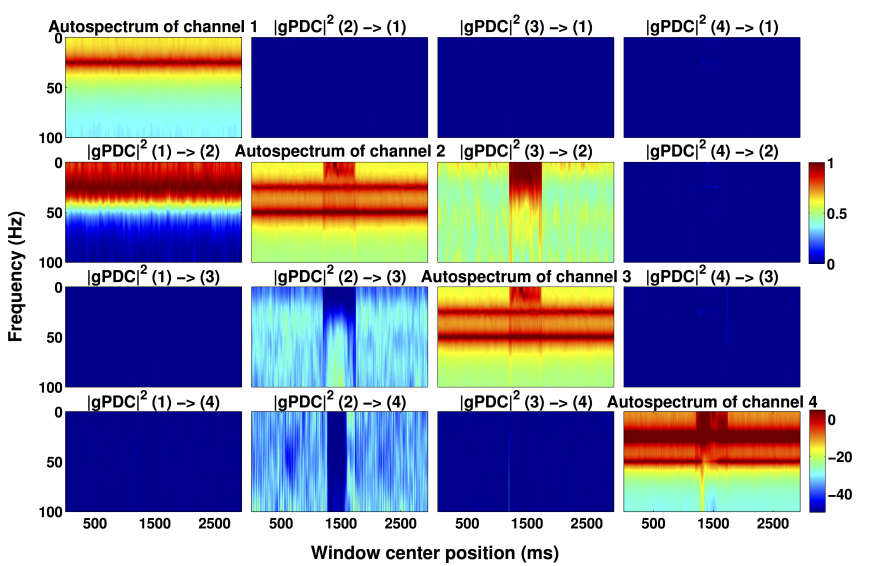

Fig. 4: Time-frequency plot of the autospectrum of each channel on the diagonals (in $\mathrm{dB}$ ). In the off-diagonals lie the estimated $g$ PDC over time.

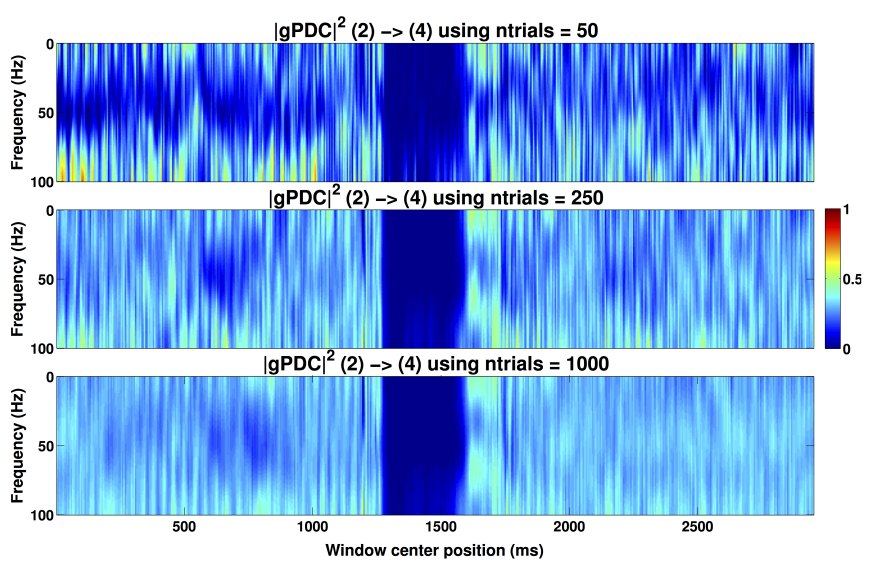

Fig. 5: Time-frequency plots of $g \mathrm{PDC}$ for increasing numbers of available trials during MVAR estimation.

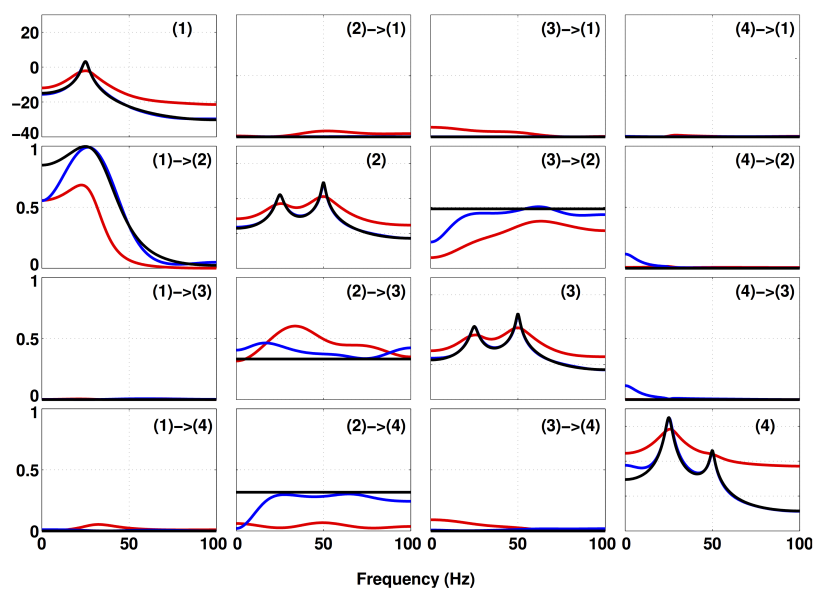

Fig. 6: Plots of the gPDC and the autospectrum (in $\mathrm{dB}$ ) when using the coefficients estimated with two kinds of algorithm: NS for segments in blue and NS stacked in red. The results using the true values of $\mathbf{A}_{p}(\cdot)$ are in black. 


\section{DISCUSSION}

We have chosen to adapt the Nuttall-Strand algorithm because it is a generalization of Burg's algorithm for spectral estimation [7] that has proven to be very efficient for autoregressive estimation in the univariate case, and because [14] has shown that the NS algorithm often outperforms LS and VM in multichannel autoregressive estimation when long records are available. Using the same considerations for the SegNS, implementing Vieira-Morf algorithm for segments (SegVM) is immediate.

Algorithm validation was based on testing two toy models. Care was taken so that the models would follow realistic ERP behaviour [13]. This kind of concern is of crucial importance and yet absent from related works in the literature.

ERPs generated via [13]'s model have an intrinsic variability in amplitude and time delays so that network structure change happen at slightly different times. Additionally, some simulated trials randomly failed to trigger network switch thereby adding a realistic feature to our simulations. This rate of ERP failure impacted MVAR estimation, since connectivity trial subpopulations coexist whereas a single estimated model is being used for both. It is the subpopulation proportions that decide whether the results point towards one network structure or another. For the examples here, the rate of switch failure was of approximately $20 \%$, and the residual post-ERP gPDC seen in Fig. 2 is due to this fact.

The results obtained in this work show that our algorithm is a viable option for studying the influence of an ERP signal on a network of neural structures. However, one should be aware that parameters like model order and window size must be appropriately tuned before performing any estimation. The results of Fig. 5 show that an increase in the number of available trials induces a decrease in $g$ PDC's time variability.

\section{FINAL COMMENTS}

We have presented a new alternative for estimating timevarying neural connectivity, using an algorithm based on the Nuttall-Strand algorithm for MVAR estimation. Simulations used physiologically-inspired toy models, portraying the intrinsic viability of our algorithm in this kind of application.

Further work is now concentrated on the statistically based comparison between the algorithms proposed here to alternatives like [15] and [9], using simulations on toy models and real EEG data. Furthermore, we intend to extend the results in [3] to the short segment case.

\section{ACKNOWLEDGMENT}

P.L.C.R gratefully acknowledges the support from the CNPq 130765/2014-7 grant and L. A. B. to the CNPq 307163/2013-0 grant.

\section{REFERENCES}

[1] O. Sporns, Networks of the Brain. The MIT Press, 2010.

[2] L. A. Baccalá and K. Sameshima, Methods in Brain Connectivity Inference through Multivariate Time Series Analysis. CRC Press, 2014, ch. Brain Connectivity, pp. 1-9.
[3] L. A. Baccala, C. S. N. de Brito, D. Y. Takahashi, and K. Sameshima, "Unified asymptotic theory for all partial directed coherence forms," Philosophical Transactions of the Royal Society A: Mathematical, Physical and Engineering Sciences, vol. 371, no. 1997, pp. $20120158-20120158$, Jul. 2013.

[4] L. A. Baccala and K. Sameshima, "Partial directed coherence: a new concept in neural structure determination," Biological cybernetics, vol. 84 , no. 6 , pp. 463-474, 2001.

[5] M. J. Kaminski and K. J. Blinowska, "A new method of the description of the information flow in the brain structures," Biological cybernetics, vol. 65 , no. 3, pp. 203-210, 1991.

[6] L. Barnett and A. K. Seth, "The MVGC multivariate Granger causality toolbox: A new approach to Granger-causal inference," Journal of Neuroscience Methods, vol. 223, pp. 50-68, 2014.

[7] S. L. Marple, Digital Spectral Analysis: With Applications (PrenticeHall Series in Signal Processing). Prentice Hall, 1987.

[8] S. J. Luck, An Introduction to the Event-Related Potential Technique (Cognitive Neuroscience). MIT Press, 2005.

[9] M. Ding, S. L. Bressler, W. Yang, and H. Liang, "Short-window spectral analysis of cortical event-related potentials by adaptive multivariate autoregressive modeling: data preprocessing, model validation, and variability assessment," Biological cybernetics, vol. 83, no. 1, pp. $35-45,2000$.

[10] S. De Waele and P. M. Broersen, "The burg algorithm for segments," Signal Processing, IEEE Transactions on, vol. 48, no. 10, pp. 2876$2880,2000$.

[11] L. A. Baccala and K. Sameshima, "Generalized partial directed coherence," in Digital Signal Processing, 2007 15th International Conference on. IEEE, 2007, pp. 163-166.

[12] H. Lutkepohl, New Introduction to Multiple Time Series Analysis. Springer, 2007.

[13] B. H. Jansen and V. G. Rit, "Electroencephalogram and visual evoked potential generation in a mathematical model of coupled cortical columns," Biological cybernetics, vol. 73, no. 4, pp. 357-366, 1995.

[14] L. S. Marple and A. H. Nuttall, "Experimental comparison of three multichannel linear prediction spectral estimators," in IEE Proceedings F (Communications, Radar and Signal Processing), vol. 130. IET, 1983, pp. 218-229.

[15] E. Moller, B. Schack, M. Arnold, and H. Witte, "Instantaneous multivariate EEG coherence analysis by means of adaptive highdimensional autoregressive models," Journal of neuroscience methods, vol. 105, no. 2, pp. 143-158, 2001. 